Therefore, $k_{m}=k_{n}$, and we have the relation

$$
p_{m}(z)=z^{m-n} p_{n}(z)=z^{m-n}\left(k_{n} z^{n}+l_{n}\right) .
$$

We have assumed, until now, that the sequence $p_{2}(0), p_{3}(0), \cdots$ contained a nonzero term. If this is not the case, the last result still holds with $n=1$, as may be seen from (4) in the same way as before.

Now we have, if $m \geqq n, m^{\prime} \geqq n, m \neq m^{\prime}$,

$$
\int_{-\pi}^{+\pi} f(\theta) e^{i\left(m-m^{\prime}\right) \theta}\left|k_{n} z^{n}+l_{n}\right|^{2} d \theta=0, \quad z=e^{i \theta} .
$$

Whence, except on a set of measure zero, we have

$$
f(\theta)=\text { const. }\left|k_{n} z^{n}+l_{n}\right|^{-2}, \quad z=e^{i \theta} .
$$

We conclude the proof with the obvious remark that the polynomials $1, z, z^{2}, \cdots, z^{n-1}$ are orthogonal on the unit circle $|z|=1$ with the weight function (11).

STANFORd UNIVERSITY

\title{
A FACTORIZATION THEOREM APPLIED TO A TEST FOR PRIMALITY*
}

D. H. LEHMER

Certain tests for primality based on the converse of Fermat's theorem and its generalizations have been devised and applied by the writer during the past ten years. $\dagger$ Perhaps the most useful test for the investigation of a large number $N$ of no special form may be given as follows: $\ddagger$

THEOREM 1. If $N$ divides $a^{N-1}-1$ but is relatively prime to $a^{(N-1) / p}$ -1 , where $p$ is a prime, then all the possible factors of $N$ are of the form $p^{\alpha} x+1$, if $N-1$ is divisible by $p^{\alpha},(\alpha \geqq 1)$.

Strictly speaking this is not a test for primality since the theorem merely gives a restriction on the factors of $N$. If $p^{\alpha}>N^{1 / 2}$ then, obviously, $N$ is a prime. If $p^{\alpha}$ is only fairly large, the theorem gives

* Presented to the Society, February 26, 1938.

$\dagger$ This Bulletin, vol. 33 (1927), pp. 327-340; vol. 34 (1928), pp. 54-56; vol. 35 (1929), pp. 349-350; vol. 38 (1932), pp. 383-384; vol. 39 (1933), pp. 105-108; Annals of Mathematics, (2), vol. 31 (1930), pp. 419-448; Journal of the London Mathematical Society, vol. 10 (1935), pp. 162-165; American Mathematical Monthly, vol. 43 (1936), pp. 347-354.

$\ddagger$ This Bulletin, vol. 33 (1927), p. 331 . 
a good restriction on the factors of $N$ and a much better restriction on the values of $u$ in the equation

$$
N=u^{2}-v^{2}=(u+v)(u-v) ;
$$

in fact $2 u \equiv N+1\left(\bmod p^{2 \alpha}\right)$. If no large divisor $p^{\alpha}$ of $N-1$ is known, it may be necessary to apply Theorem 1 several times and to combine the several restrictions thus obtained. Finally if no divisor $p^{\alpha}$ (other than the trivial factor $p^{\alpha}=2$ ) of $N-1$ is known, then Theorem 1 tells us nothing at all.

It thus appears that the problem of proving $N$ a prime is closely allied to the factorization of $N-1$. In general it is impossible to say anything about the factors of $N-1$ beyond the obvious remark that $N-1$ is even. In the interesting case where $N$ is a divisor of a number of the form $y^{n}-1$, it is possible to make the factorization of $N-1$ depend upon the factors* of $y^{k}-1$, where $k<n$. It is the purpose of this note to indicate how this may be done.

To be more specific, let $n$ be a positive integer, and let $Q_{n}(x)$ $=x^{\phi}+\cdots$ be the irreducible polynomial whose roots are the primitive $n$th roots of unity, so that we have the familiar factorization

$$
y^{n}-1=\prod_{\delta \mid n} Q_{\delta}(y),
$$

where $\delta$, as indicated, ranges over all the divisors of $n$. Then the factorization of numbers of the form $y^{n}-1$ depends on the factorization of $N=Q_{n}(y)$. If we suspect that $N$ is a prime, it may be tested for primality provided something is known of the factors of $N-1$. Before discussing this topic, we need two lemmas.

LEMma 1. $\dagger$ If $n=s d$, where $s$ is the product of all distinct prime factors of $n$, and $d \geqq 1$, then

$$
Q_{n}(y)=Q_{s}\left(y^{d}\right) .
$$

Lemma 2. If $n$ is not divisible by the prime $q$, then

$$
Q_{n q}(y)=Q_{n}\left(y^{q}\right) / Q_{n}(y) \text {. }
$$

Both lemmas can be made to follow easily from the familiar $\ddagger$ Dedekind inversion of (1):

* See Cunningham and Woodall, Factorization of $y^{n} \pm 1$, London, 1925, for extensive tables for $y \leqq 12$. For $y>12$, see Cunningham, Messenger of Mathematics, vol. 57 (1927), pp. 72-80; see also Kraitchik, Recherches sur la Théorie des Nombres, vol. 2, Paris, 1929, pp. 84-159.

$\dagger$ Trudi, Annali di Matematica, (2), vol. 2 (1868-1869), pp. 160-162.

$\ddagger$ Journal für die reine und angewandte Mathematik, vol. 54 (1857), pp. 25-26. 


$$
Q_{n}(y)=\prod_{\delta \mid n}\left(y^{n / \delta}-1\right)^{\mu(\delta)},
$$

where $\mu$ is the Möbius function defined by $\mu(1)=1$, and for $k>1$ by $\mu(k)=(-1)^{h}$ or zero according as $k$ is a product of $h$ distinct primes or not.

To prove Lemma 1 , we note that $\mu(\delta)=0$, except when $\delta$ is a divisor of $s$. Therefore (4) may be written

$$
Q_{n}(y)=\prod_{\delta / \delta}\left\{\left(y^{d}\right)^{s / \delta}-1\right\}^{\mu(\delta)}=Q_{s}\left(y^{d}\right) .
$$

Lemma 2 may be established by noting that the divisors of $n q$ are of the forms $\delta$ and $q \delta$, where $\delta$ ranges over the divisors of $n$, so that (4) becomes in this case

$$
Q_{n q}(y)=\prod_{\delta \mid n}\left(y^{n q / \delta}-1\right)^{\mu(\delta)} \prod_{\delta \mid n}\left(y^{n / \delta}-1\right)^{\mu(q \delta)} .
$$

Since $q$ does not divide $\delta, \mu(q \delta)=-\mu(\delta)$, and we obtain Lemma 2 at once on comparing (5) with (4).

Returning to the problem of factoring $N-1=Q_{n}(y)-1$ we find that three cases present themselves according as $n$ has 0,1 , or more than one odd prime factor.

Case 1. If $n=2^{\lambda}$, we exclude as trivial the cases $n=1$ and 2 in which $Q_{1}(y)=y-1$, and $Q_{2}(y)=y+1$. For $n=2^{\lambda},(\lambda>0)$, (4) gives

$$
N=Q_{n}(y)=\left(y^{n}-1\right) /\left(y^{n / 2}-1\right)=y^{n / 2}+1 ;
$$

so the factorization of $N-1$ is obvious. As a matter of fact, when $y$ is odd $N$ is oddly even, and we are really concerned with $N / 2$ and hence with factoring

$$
(N / 2)-1=\frac{1}{2}\left(y^{n / 2}-1\right)=\frac{1}{2}(y-1)(y+1)\left(y^{2}+1\right) \cdots\left(y^{n / 4}+1\right) .
$$

The decomposition of these binomials into their prime factors may be thought of as known, since their exponents are all considerably less than $n / 2$.

Case 2. Assume that $n=2^{\lambda} q^{\beta}, \lambda \geqq 0, q$ an odd prime, $\beta \geqq 1$. In case $\lambda=0$, formula (4) gives

$$
N=Q_{n}(y)=\left(y^{n}-1\right) /\left(y^{n / q}-1\right) .
$$

Hence

$$
N-1=y^{n / q}\left(y^{\phi}-1\right) /\left(y^{n / q}-1\right),
$$

where $\phi=\phi(n)=n-n / q=q^{\beta-1}(q-1)$. In case $\lambda>0$, (4) gives 
1939]

$$
N=Q_{n}(y)=\left(y^{n / 2}+1\right) /\left(y^{n / 2 q}+1\right) .
$$

Hence

$$
N-1=y^{n / 2 q}\left(y^{\phi}-1\right) /\left(y^{n / 2 q}+1\right)
$$

where, in this case,

$$
\phi=\phi(n)=(n / 2)-(n / 2 q)=2^{\lambda-1} q^{\beta-1}(q-1) .
$$

In either case, then, the factorization of $N-1$ can be made to depend on that of binomials of much lower degree. For example, if $n=200$, $N-1=Q_{200}(y)-1=y^{20}\left(y^{80}-1\right) /\left(y^{20}+1\right)=y^{20}\left(y^{40}+1\right)\left(y^{20}-1\right)$.

Case 3. Assume that $n$ has more than one odd prime factor. The final case is much more complicated; it is no longer true that all the prime factors of $N-1$ divide either $y$ or $y^{m}-1$ for $m<n$. However, it is possible to account in this way for some of the factors of $N-1$ by the following theorem:

THEOREM 2. Let $N=Q_{n}(y)$, and write $n=d s=q t d$, where $s$ is the product of the distinct prime factors of $n$ and $q$ is any prime factor of $N$; then $N-1$ is divisible by $y^{d}$ and also by $y^{d(q-1)}-1$, unless $t$ divides $q-1$ in which case $N-1$ is divisible by the integer $\left(y^{d(q-1)}-1\right) / Q_{t}\left(y^{d}\right)$.

PROOF. It is well known that for every $s>1, Q_{s}(0)=1$. In other words $Q_{s}(x)-1$ is divisible by $x$. Hence by Lemma 1

$$
N-1=Q_{n}(y)-1=Q_{s}\left(y^{d}\right)-1
$$

is divisible* by $y^{d}$. By Lemma 2, replacing $n$ and $y$ by $t$ and $y^{d}$, respectively, we may write, in view of (6),

$$
N-1=Q_{t q}\left(y^{d}\right)-1=\frac{Q_{t}\left(y^{d q}\right)-Q_{t}\left(y^{d}\right)}{Q_{t}\left(y^{d}\right)} .
$$

The polynomial $Q_{t}\left(y^{d q}\right)-Q_{t}\left(y^{d}\right)$ is divisible by $y^{d q}-y^{d}$ and hence by $y^{d(q-1)}-1$. In fact, if $f(x)=a_{0}+a_{1} x+a_{2} x^{2}+\cdots$ is any polynomial, every term of the difference

$$
f(u)-f(v)=a_{1}(u-v)+a_{2}\left(u^{2}-v^{2}\right)+a_{3}\left(u^{3}-v^{3}\right)+\cdots
$$

is divisible by $u-v$. Now since (7) is an identity in $y^{d}$, we may replace $y^{d}$ by $z$ and write

$$
Q_{t}\left(z^{q}\right)-Q_{t}(z)=z\left(z^{q-1}-1\right) P(z),
$$

\footnotetext{
* Incidentally, $N-1$ does not contain a higher power of $y$ than $y^{d}$ since the penultimate term of $Q_{s}(x)$ is $-\mu(s) x= \pm x$.
} 
where $P(z)$ is a polynomial with integral coefficients. But the right member of (8) is algebraically divisible by $Q_{t}(z)$; and since $Q_{t}(z)$ is irreducible it must divide either $z^{q-1}-1$ or else $P(z)$. By (1) it follows that $z^{q-1}-1$ is divisible by $Q_{t}(z)$ if and only if $t$ divides $q-1$. Hence the theorem follows at once.

The algebraic factorization of $Q_{n}(y)-1$ under Case 3 may be given for a few values of $n$. By Lemma 1, we may confine ourselves to those values of $n$ which are products of distinct primes and since, if $k$ is odd,

$$
Q_{2 k}(x)=Q_{k}(-x),
$$

we take only odd values of $n$ which have no square factors. For the first five such values we have

$$
\begin{aligned}
& Q_{15}(y)-1=y\left(y^{4}-1\right)\left(y^{3}-y^{2}+1\right), \\
& Q_{21}(y)-1=y(y-1)\left(y^{3}+1\right)\left(y^{7}+y+1\right), \\
& Q_{33}(y)-1=y\left(y^{10}-1\right)\left(y^{9}-y^{8}+y^{6}-y^{5}+y^{3}-y^{2}+1\right), \\
& Q_{35}(y)-1=y\left(y^{2}+1\right)\left(y^{6}-1\right)\left(y^{15}-y^{14}-y^{13}+y^{12}+y^{11}\right. \\
& \left.-y^{9}+y^{5}-y^{2}+1\right) \text {, } \\
& Q_{55}(y)-1=y(y-1)\left(y^{2}+1\right)\left(y^{5}+1\right)\left(y^{31}-y^{29}+y^{27}-y^{25}\right. \\
& +y^{23}+y^{20}-y^{18}+y^{16}-y^{14}+y^{12}+y^{11} \\
& +y+1) \text {. }
\end{aligned}
$$

The use of this table is illustrated by the following examples.

Let $N=Q_{60}(11)=46329453543600481$. In view of Lemma 1 and (9), we have

$$
Q_{60}(11)=Q_{30}\left(11^{2}\right)=Q_{15}\left(-11^{2}\right) .
$$

Substituting $y=-11^{2}$ in (10) we find

$$
N-1=11^{2}\left(11^{8}-1\right)\left(11^{6}+11^{4}-1\right),
$$

from which we easily obtain the decomposition into primes

$$
N-1=2^{5} \cdot 3 \cdot 5 \cdot 11^{2} \cdot 61 \cdot 7321 \cdot 1786201 .
$$

Choosing $p=1786201$ for the application of Theorem 1 we find that

$$
2^{(N-1) / p}-1 \equiv 25378134441594865=A-1(\bmod N)
$$

and that $A-1$ is prime to $N$. Furthermore we find that

$$
A^{p}=2^{N-1} \equiv 1(\bmod N) \text {. }
$$


Hence by Theorem 1 the factors of $N$ are of the form $1786201 x+1$. Combining* this with $60 x+1$ we get a restriction on the factors of $N$ of the form $107172060 x+1$. Only two numbers of this form exist below $N^{1 / 2}$, namely 107172061 and 214344121 . Since neither of these is a factor of $N$, it follows that $N$ is a prime.

As a second example consider

$$
N=Q_{240}(2)=18518800563924107521 .
$$

By Lemma 1 and (9) we have

$$
N=Q_{240}(2)=Q_{30}\left(2^{8}\right)=Q_{15}\left(-2^{8}\right) .
$$

Hence by (10)

$N-1=2^{8}\left(2^{32}-1\right)\left(2^{24}+2^{16}-1\right)=2^{8} \cdot 3 \cdot 5 \cdot 17 \cdot 257 \cdot 65537 \cdot 16842751$,

the last factor being easily identified as a prime. $\dagger$ Armed with this complete factorization of $N-1$ we are now in a position to apply Theorem 1 to the number $N$, which has been investigated and declared prime by M. Kraitchik $\ddagger$ in 1929 by a plausible though nonrigorous method.§ It turns out however that the first part of the hypothesis of Theorem 1 is not satisfied, that is, $N$ does not divide $11^{N-1}-1$. Hence, by Fermat's theorem, $N$ is composite. To settle this question we applied a new type of machine for combining linear congruences to the problem of factoring $N$ with the result that

$$
N=394783681 \cdot 46908728641 .
$$

LEHIGH UNIVERSITY

* Every factor of $Q_{n}(y)$ not dividing $n$ is of the form $n x+1$.

$\dagger$ By the method given in the American Mathematical Monthly, vol. 43 (1936), pp. 347-354.

$\ddagger$ Recherches sur la Theorie des Nombres, vol. 2, Paris, 1929, pp. 12-17.

$\S$ This Bulletin, vol. 36 (1930), pp. 847-850. 